\title{
Fatores associados à falta de diversidade alimentar no segundo semestre de vida
}

\author{
Factors associated with lack of dietary diversity in the \\ second semester of life
}

\author{
Maria Inês Couto de Oliveira ${ }^{1}$, Renata Ribeiro Rigotti², Cristiano Siqueira Boccolini ${ }^{3}$
}

\begin{abstract}
Resumo
Objetivo: Investigar os fatores associados à falta de alimentação diversificada no segundo semestre de vida, pois a Organização Mundial da Saúde preconiza a introdução de alimentos variados complementares ao leite materno a partir dos 6 meses. Métodos: Foram entrevistadas acompanhantes de 580 crianças que compareceram à Campanha de Vacinação de 2006 em município urbano da região Sudeste do Brasil. O desfecho "alimentação não diversificada" correspondeu à ingestão de menos de cinco grupos de alimentos (carnes, leite, frutas, legumes e feijão) nas últimas 24 horas. As variáveis associadas ao desfecho ( $p \leq 0,20)$ na análise bivariada foram selecionadas para análise multivariada. Razões de prevalência ajustadas foram obtidas pelo modelo de regressão de Poisson $(p \leq 0,05)$. Resultados: Do total, $64,5 \%$ das crianças não recebiam alimentos diversificados. Na análise múltipla, a criança não estar acompanhada pela mãe $(R P=1,170)$ e a internação prévia $(R P=1,214)$ foram fatores diretamente associados à falta de diversidade alimentar. $O$ nascimento em hospital privado $(R P=0,816)$ e a idade da criança crescente em dias $(\mathrm{RP}=0,997)$ se associaram inversamente ao desfecho. Conclusões: Apesar de sua importância para a nutrição infantil, a alimentação diversificada no segundo semestre de vida vem sendo pouco praticada. Os fatores associados identificados devem ser considerados no âmbito das políticas públicas de nutrição e de saúde.
\end{abstract}

Palavras-chave: diversidade; consumo de alimentos; alimentação complementar; nutrição infantil; estudos transversais.

\begin{abstract}
Objective: to study factors associated with the lack of dietary diversity in the second semester of life considering that World Health Organization recommends the introduction of healthy and diversified complementary food to breast milk since the sixth month of life. Methods: we interviewed children caregivers $(n=580)$ who attended the 2006 Vaccination Campaign in an urban municipality in southeastern Brazil. The outcome "lack of dietary diversity" corresponded to the intake of less than five food groups: meat, milk, fruits, vegetables and beans in the last 24 hours. Variables associated with the outcome $(p \leq 0.20)$ in bivariate analysis were selected for multivariate analysis. Adjusted prevalence ratios were obtained by Poisson regression model ( $\mathrm{p} \leq 0.05)$. Results: a total of $64.5 \%$ of the children experienced lack of dietary diversity. In multivariate analysis, absence of direct care from the mother $(\mathrm{OR}=1.170)$ and previous hospitalization $(\mathrm{OR}=1.214$ ) were factors directly associated with lack of dietary diversity. Birth in private hospital $(\mathrm{OR}=0.816)$ and greater age in days of the child $(\mathrm{OR}=0.997)$ were inversely associated with the outcome. Conclusions: despite the importance for infant nutrition, complementary diversified diet was not usually provided to children aged 6-11 months. The identified associated factors should be considered in health and nutrition public policies.
\end{abstract}

Keywords: diversity; food consumption; supplementary feeding; infant nutrition; cross-sectional studies.

\footnotetext{
1Departamento de Epidemiologia e Bioestatística, Instituto de Saúde Coletiva, Universidade Federal Fluminense (UFF) - Niterói (RJ), Brasil.

${ }^{2}$ Programa de Pós-graduação em Saúde Coletiva, Universidade Federal Fluminense (UFF) - Niterói (RJ), Brasil.

3Laboratório de Informação em Saúde, Instituto de Comunicação e Informação Científica e Tecnológica em Saúde, Fundação Oswaldo Cruz (FIOCRUZ) - Rio de Janeiro (RJ), Brasil.

Trabalho realizado no Institudo de Saúde Coletiva (ISC), Universidade Federal Fluminense (UFF) - Niterói (RJ), Brasil.

Endereço para correspondência: Maria Inês Couto de Oliveira - Departamento de Epidemiologia e Bioestatística, Instituto de Saúde Coletiva, Universidade Federal Fluminense (UFF), Rua Marques de Paraná, 303, $3^{\circ}$ andar, Prédio anexo - Centro - CEP: 24033-900 - Niterói (RJ), Brasil - Email: marinesco@superig.com.br

Fonte de financiamento: nenhuma.
}

Conflito de interesses: nada a declarar. 


\section{INTRODUÇÃO}

Aos 6 meses de vida, as crianças amamentadas devem começar a receber alimentos complementares ao leite materno que sejam nutricionalmente adequados para a prevenção da anemia, da desnutrição, do sobrepeso e da morbimortalidade na infância ${ }^{1}$. A Organização Mundial da Saúde (OMS) recomenda que essa alimentação seja oportuna (introduzida quando as necessidades de energia e nutrientes ultrapassam o que o aleitamento materno exclusivo pode oferecer), adequada (proporcionando energia, proteínas e micronutrientes suficientes para satisfazer às necessidades nutricionais da criança), inócua (preparo e armazenamento de forma higiênica, oferecidos com mãos e utensílios limpos) e com consistência e frequência adequadas à idade, para que atendam às sensações de fome e saciedade da criança, satisfazendo às suas necessidades nutricionais ${ }^{2}$.

Tanto a introdução precoce da alimentação complementar como a tardia podem trazer prejuízos à saúde da criança. A OMS preconiza o aleitamento materno exclusivo por seis meses e o complementado por alimentos saudáveis e diversificados até os 2 anos de idade ou mais ${ }^{3}$. Um conjunto de indicadores foi criado pela OMS para avaliar as práticas de alimentação infantil ${ }^{4}$, dos quais um deles é a diversidade mínima da dieta, permitindo, assim, avaliar a situação atual das práticas de alimentação complementar, incluindo a variação alimentar ${ }^{5}$. Este artigo teve como objetivo investigar a prevalência de alimentação não diversificada no segundo semestre de vida, bem como analisar os fatores associados a essa prática.

\section{MÉTODOS}

Estudo transversal conduzido mediante entrevista com acompanhantes de crianças menores de 1 ano no dia da Campanha Nacional de Vacinação de 2006, quando foi realizado levantamento de hábitos alimentares infantis em Barra Mansa, município do Sudeste do Brasil, com população estimada em 179 mil habitantes residentes em área urbana ${ }^{6}$. Esta pesquisa foi baseada no Projeto Amamentação e Municípios (AMAMUNIC), cujo objetivo foi monitorar as práticas de alimentação infantil no Brasil 7 .

Os planos amostrais do inquérito foram elaborados com base em informações fornecidas pela Secretaria de Saúde de Barra Mansa, relativas ao número de postos de vacinação em cada distrito sanitário e à estimativa do número de crianças menores de 1 ano que seriam vacinadas em cada um dos locais. Considerando que as crianças não estavam distribuídas uniformemente nos postos de vacinação (conglomerados), adotou-se o sorteio em dois estágios, com probabilidade proporcional ao tamanho dos conglomerados. No primeiro estágio, foram sorteados os postos de vacinação, e, no segundo, as crianças em cada um deles, de forma sistemática. A amostra desenvolvida é considerada equiprobabilística ou autoponderada, ou seja, todas as crianças que foram aos postos de vacinação durante as campanhas tinham a mesma probabilidade de pertencer à amostra sorteada, evitando a necessidade de posterior ponderação ${ }^{7}$.

Em 2006, o tamanho amostral para a pesquisa em Barra Mansa foi calculado em 1.140 crianças de até 1 ano. Foram entrevistadas 1.287 mães ou responsáveis de crianças menores de um ano, em 36 postos de vacinação, mas foram excluídas aquelas com menos de 6 meses de vida. Assim, obteve-se um número de 580 crianças com idade entre 6 e 12 meses, além de 1.287 mães ou responsáveis entrevistadas. Nesse ano, a cobertura vacinal, segundo o Setor de Epidemiologia da Secretaria Municipal de Saúde, foi de $96 \%$. Dessa forma, essa subamostra foi suficiente para detectar diferenças de 3\%, com um nível de significância de $5 \%$ e poder de teste de $80 \%$, calculado a posteriori.

A análise por extratos etários tem sido um procedimento usual em estudos fundamentados em inquéritos conduzidos sob a metodologia AMAMUNIC ${ }^{7}$. Na presente pesquisa, as crianças no segundo semestre de vida corresponderam a quase $50 \%$ daquelas menores de 1 ano, reforçando, assim, a hipótese de representatividade dessa faixa etária na amostra total.

As entrevistas foram realizadas por agentes comunitários de saúde e por estudantes de graduação de enfermagem, sob a supervisão da coordenadora da Área Técnica de Alimentação e Nutrição. Os entrevistadores foram treinados para o uso do questionário de coleta de dados de forma padronizada para abordagem aos acompanhantes.

Para a seleção das crianças na fila de vacinação, foi aplicado o sorteio sistemático. Antes da entrevista, as mães eram informadas sobre a pesquisa e sobre a não obrigatoriedade de participação. Visando à padronização das informações necessárias ao esclarecimento das mães ou acompanhantes a respeito do caráter da pesquisa, foi mostrado um Termo de Apresentação, do qual não se obrigava a assinatura para não interferir no andamento das atividades rotineiras de vacinação. $\mathrm{O}$ instrumento só foi aplicado mediante o consentimento verbal do responsável pela criança, respeitando-se o direito de recusa e interrupção da entrevista, além de garantir o sigilo das informações.

O questionário padronizado incluía perguntas sobre as práticas de alimentação nas últimas 24 horas - o consumo de leite materno, água, chá, fruta, suco de fruta, outros tipos de leite, mingau, sopa/papa de legumes e "comida de panela" - e se os alimentos continham carne ou feijão, seguindo o questionário padronizado pelo Ministério da Saúde, conforme a metodologia AMAMUNIC ${ }^{7}$. Foram coletadas também informações sobre o perfil socioeconômico e reprodutivo das mães biológicas e características do nascimento e de assistência à criança.

Inicialmente, caracterizou-se o perfil das mães e das crianças, além de descrever as práticas alimentares destas no segundo semestre de vida. A diversidade alimentar foi um indicador 
adaptado do proposto pela OMS, constituído, originalmente, por sete grupos de alimentos: 1. grãos, tubérculos e raízes; 2. leguminosas e nozes; 3. produtos lácteos (leite, iogurte e queijo); 4. carnes (carne, peixe, aves e miúdos); 5. ovos; 6. frutas e vegetais ricos em vitamina A; 7. outras frutas e legumes 4 . No presente estudo, a diversidade alimentar foi caracterizada como o consumo de cinco grupos de alimentos nas últimas 24 horas: leite (materno ou outro), carnes, frutas, legumes e feijão. Essa adaptação foi necessária, pois o questionário padronizado não contemplava todos os grupos alimentares propostos pela OMS, e foi previamente utilizada por Oliveira et al. ${ }^{8}$. O desfecho "ausência de diversidade alimentar" foi tratado de forma binária, com as respostas categorizadas em: $0=$ a criança recebeu os cinco grupos de alimentos pelo menos uma vez nas últimas 24 horas [referência]; 1 = a criança recebeu zero a quatro desses grupos de alimentos.

Foram explorados fatores independentes, como acompanhante da criança, idade, escolaridade e trabalho maternos, paridade, município de moradia, área do local de vacinação, financiamento do hospital de nascimento, tipo de parto, sexo e idade do bebê, peso ao nascer, ocorrência de internações anteriores, uso de mamadeira e de chupeta. Foi verificada também a associação entre os fatores de exposição e o desfecho "ausência de diversidade alimentar".

Primeiramente, foi desenvolvida a análise univariada para verificação da distribuição das variáveis independentes e do desfecho sob investigação; em seguida, fez-se a análise bivariada entre cada variável independente e o desfecho. Foram realizados testes de hipóteses de qui-quadrado e obtidas razões de prevalência (RP) brutas com seus respectivos intervalos de confiança de 95\% (IC95\%). Variáveis independentes, que, na análise bivariada, mostraram-se associadas ao desfecho com nível de significância observado menor ou igual a $20 \%$ no teste de qui-quadrado ( $\mathrm{p}$-valor $\leq 0,20$ ), foram selecionadas para análise múltipla.

As RP ajustadas relativas ao desfecho foram obtidas por modelo de regressão de Poisson com variância robusta pela prevalência elevada do desfecho ${ }^{9}$. O modelo final, utilizado para estimar medidas de associação com seus respectivos IC95\%, foi composto pelas variáveis independentes que obtiveram $\mathrm{p}$-valor $\leq 0,05$. A análise dos dados foi realizada pelo software SPSS 17.0.

A pesquisa original AMAMUNIC foi submetida ao Comitê de Ética da Faculdade de Saúde Pública (COEP) para avaliação quanto a riscos para seres humanos, de acordo com a Resolução CNS n ${ }^{\circ} 196 / 96^{10}$, e aprovada em sua 7a/01 Sessão Ordinária. $\mathrm{O}$ estudo presente utilizou um banco de dados secundários, sem possibilidade de identificação dos acompanhantes e das crianças. Esse uso foi autorizado pela Coordenação da Área Técnica de Alimentação e Nutrição da Secretaria de Saúde de Barra Mansa.

\section{RESULTADOS}

No segundo semestre de vida, $35,5 \%$ das 580 crianças receberam alimentação diversificada, com menor frequência $(22,9 \%)$ na faixa etária de 6 a 7 meses, atingindo 39,3\% de 8 a 9 meses e $42,3 \%$ de 10 a 11 meses.

Das entrevistadas, $83,4 \%$ eram mães que estavam acompanhando seus filhos na fila de vacinação, $61 \%$ não tinham concluído o segundo grau, $25,1 \%$ estavam trabalhando, $94,5 \%$ residiam no meio urbano, $52,1 \%$ eram primíparas, $62,2 \%$ tiveram seus filhos em hospital privado e $65,6 \%$ foram submetidas a parto cesáreo. Além disso, 57,3\% das crianças recebiam água ou alimentos por mamadeira $(57,3 \%)$ e $7,3 \%$ já haviam sido internadas por alguma patologia (Tabela 1).

Apenas $60,2 \%$ das crianças estavam sendo amamentadas, mas $99,7 \%$ recebiam produtos lácteos. Entre as lactentes, $46,4 \%$ também tomavam outro leite e $36,4 \%$ comiam mingau. Ademais, $78,7 \%$ consumiam sopa/papa de legumes, $77,8 \%$, feijão, e $84,7 \%$, frutas ou suco de frutas, enquanto que $48,4 \%$ recebiam comida de panela. Menos de $60 \%$ das crianças comiam carne $(59,2 \%)$ em sopas/papas ou na comida de panela (Tabela 2).

$\mathrm{Na}$ análise bivariada, apresentaram-se como variáveis diretamente associados à ausência de alimentação diversificada: a criança não estar acompanhada pela mãe, a escolaridade materna inferior ao segundo grau completo, o trabalho materno, o baixo peso ao nascer, a criança ter idade de 6 a 8 meses e ter sido internada previamente por alguma patologia. O nascimento em hospital privado, o parto cesáreo e o sexo feminino apresentaram-se inversamente associados à falta de diversidade alimentar (Tabela 3).

$\mathrm{Na}$ análise múltipla, foram confirmados como fatores diretamente associados ao desfecho: a criança não estar acompanhada pela mãe $(\mathrm{RP}=1,17)$ e a internação prévia $(\mathrm{RP}=1,21)$. Já os inversamente associados foram: o nascimento em hospital privado $(\mathrm{RP}=0,82)$ e a idade crescente em dias $(\mathrm{RP}=0,99)$ (Tabela 4$)$.

\section{DISCUSSÃO}

Os padrões de alimentação observados no segundo semestre de vida em Barra Mansa não correspondem aos preconizados pela $\mathrm{OMS}^{4}$, pois quase dois terços das crianças não estavam recebendo alimentação diversificada, cuja ingestão é permitida aos 6 meses vida por causa do desenvolvimento fisiológico e comportamental do bebê $\hat{e}^{11}$. Para alcançar as necessidades nutricionais, é fundamental a diversidade alimentar, uma vez que quanto menor ela for, menos adequada é a ingestão de micronutrientes e pior o estado nutricional das crianças entre 6 e 24 meses de idade ${ }^{12}$.

O comportamento alimentar da criança pode ser determinado pela interação dela com o alimento, por fatores emocionais, psicológicos, socioeconômicos e culturais. Um estudo de Ramos e Stein $(2000)^{13}$ revela que os pais focalizam mais a quantidade 
Tabela 1. Características sociodemográficas e reprodutivas das mães e dados de nascimento e de assistência à criança no segundo semestre de vida, em Barra Mansa, Sudeste do Brasil, 2006

\begin{tabular}{|c|c|c|c|}
\hline Variável & Categorias & $\mathbf{n}$ & $\%$ \\
\hline \multirow[t]{2}{*}{ Acompanhante da criança } & Mãe & 484 & 83,4 \\
\hline & Outro & 96 & 16,6 \\
\hline \multirow[t]{3}{*}{ Idade da mãe } & $<20$ anos & 72 & 12,4 \\
\hline & 20 a 34 anos & 425 & 73,3 \\
\hline & $>34$ anos & 68 & 11,7 \\
\hline \multirow[t]{2}{*}{ Escolaridade } & Até $2^{\circ}$ grau incompleto & 341 & 61,0 \\
\hline & $2^{\circ}$ grau completo ou mais & 218 & 39,0 \\
\hline \multirow[t]{2}{*}{ Trabalho materno atual } & Sim & 145 & 25,1 \\
\hline & Não & 433 & 74,9 \\
\hline \multirow[t]{2}{*}{ Município de moradia } & Barra Mansa & 556 & 95,9 \\
\hline & Outro município & 24 & 4,1 \\
\hline \multirow[t]{2}{*}{ Área de moradia } & Urbana & 548 & 94,5 \\
\hline & Rural & 32 & 5,5 \\
\hline \multirow[t]{2}{*}{ Paridade } & Primípara & 302 & 52,1 \\
\hline & Multípara & 278 & 47,9 \\
\hline \multirow[t]{2}{*}{ Hospital de nascimento } & Privado & 354 & 62,2 \\
\hline & Público & 215 & 37,8 \\
\hline \multirow[t]{2}{*}{ Tipo de parto } & Normal & 199 & 34,4 \\
\hline & Cesariana & 380 & 65,6 \\
\hline \multirow[t]{2}{*}{ Sexo do bebê } & Masculino & 296 & 51,0 \\
\hline & Feminino & 284 & 49,0 \\
\hline \multirow[t]{2}{*}{ Peso ao nascer } & $<2.500 \mathrm{~g}$ & 44 & 7,7 \\
\hline & $2.500 \mathrm{~g}$ ou mais & 527 & 92,3 \\
\hline \multirow[t]{2}{*}{ Idade da criança } & 6 a 8 meses & 265 & 45,7 \\
\hline & 9 a 11 meses & 315 & 54,3 \\
\hline \multirow[t]{2}{*}{ Internação hospitalar prévia } & Sim & 42 & 7,3 \\
\hline & Não & 531 & 92,7 \\
\hline \multirow[t]{2}{*}{ Uso de chupeta } & Sim & 274 & 47,2 \\
\hline & Não & 306 & 52,8 \\
\hline \multirow[t]{2}{*}{ Uso de mamadeira } & Sim & 331 & 57,3 \\
\hline & Não & 247 & 42,7 \\
\hline Total & & 580 & 100 \\
\hline
\end{tabular}

Tabela 2. Líquidos e alimentos consumidos nas últimas 24 horas pela criança no segundo semestre de vida, em Barra Mansa, Sudeste do Brasil, 2006

\begin{tabular}{lcc}
\multicolumn{1}{c}{ Variável } & n & \% \\
Água & 559 & 96,5 \\
Chá & 98 & 17,0 \\
Leite materno & 348 & 60,2 \\
Outro leite & 386 & 67,0 \\
Mingau & 322 & 55,8 \\
Produto lácteo & 577 & 99,7 \\
Suco de fruta & 391 & 68,6 \\
Fruta & 404 & 70,5 \\
Suco ou fruta & 481 & 84,7 \\
Legumes & 454 & 78,7 \\
Carne & 342 & 59,2 \\
Feijão & 449 & 77,8 \\
Comida de panela & 279 & 48,4 \\
Total & 580 & 100 \\
\hline
\end{tabular}

dos alimentos oferecidos do que a qualidade e a formação de hábitos saudáveis de alimentação, o que pode explicar a relativa baixa prevalência de diversidade alimentar.

O ambiente doméstico, o estilo de vida dos pais e as relações interfamiliares podem ter grande influência nas preferências alimentares e afetar o equilíbrio energético da alimentação pela disponibilidade e composição dos alimentos. Assim, a família poderá estabelecer o aprendizado de hábitos socialmente aceitos ou inserir outros novos, contribuindo para a formação de um padrão de comportamento alimentar adequado ou não ${ }^{14}$.

No contexto brasileiro, foi encontrado apenas um estudo que investigou a tendência temporal do indicador "diversidade alimentar". Os dados disponíveis sobre a alimentação complementar praticada no Brasil, em geral, vêm de pesquisas advindas de inquéritos conduzidos com a metodologia AMAMUNIC ${ }^{7}$, as quais verificam o consumo individual de alimentos como frutas, suco de frutas, legumes, carne e feijão. 
Tabela 3. Prevalência e razão de prevalência bruta $(\mathrm{RPb})$ da ausência de diversidade alimentar no segundo semestre de vida, de acordo com as características sociodemográficas e reprodutivas das mães e com os dados de nascimento e de assistência à criança, em Barra Mansa, Sudeste do Brasil, 2006

\begin{tabular}{|c|c|c|c|c|}
\hline Características & $\%$ & $\mathbf{R} \mathbf{P}_{\mathrm{b}}$ & IC95\% & valor de $p$ \\
\hline \multicolumn{5}{|l|}{ Acompanhante da criança } \\
\hline Mãe & 62,9 & 1 & & \\
\hline Outro & 73,1 & 1,163 & $1,010-1,339$ & 0,036 \\
\hline \multicolumn{5}{|l|}{ Idade da mãe } \\
\hline$<20$ anos & 70,8 & 1,147 & $0,903-1,456$ & 0,260 \\
\hline 20 a 34 anos & 60,4 & 1,037 & $0,849-1,267$ & 0,722 \\
\hline$>34$ anos & 61,8 & 1 & & \\
\hline \multicolumn{5}{|l|}{ Escolaridade materna } \\
\hline Até $2^{\circ}$ grau incompleto & 67,0 & 1,130 & $0,989-1,291$ & 0,073 \\
\hline $2^{\circ}$ grau completo ou mais & 59,3 & 1 & & \\
\hline \multicolumn{5}{|l|}{ Trabalho materno atual } \\
\hline Sim & 66,3 & 1,128 & $0,968-1,315$ & 0,122 \\
\hline Não & 58,7 & 1 & & \\
\hline \multicolumn{5}{|l|}{ Local de moradia } \\
\hline Barra Mansa & 64,5 & 1 & & \\
\hline Outro município & 65,2 & 1,011 & $0,746-1,372$ & 0,943 \\
\hline \multicolumn{5}{|l|}{ Local de vacinação } \\
\hline Área urbana & 64,1 & 1 & & \\
\hline Área rural & 71,9 & 1,121 & $0,895-1,405$ & 0,319 \\
\hline \multicolumn{5}{|l|}{ Paridade } \\
\hline Primípara & 62,5 & 0,935 & $0,829-1,055$ & 0,278 \\
\hline Multípara & 66,8 & 1 & & \\
\hline \multicolumn{5}{|l|}{ Hospital de nascimento } \\
\hline Sistema Único de Saúde & 72,3 & 1 & & \\
\hline Privado & 60,4 & 0,835 & $0,742-0,941$ & 0,003 \\
\hline \multicolumn{5}{|l|}{ Tipo de parto } \\
\hline Normal & 69,4 & 1 & & \\
\hline Cesariana & 61,9 & 0,892 & 0,790-1,008 & 0,067 \\
\hline \multicolumn{5}{|l|}{ Sexo do bebê } \\
\hline Feminino & 61,7 & 0,918 & $0,812-1,037$ & 0,167 \\
\hline Masculino & 67,2 & 1 & & \\
\hline \multicolumn{5}{|l|}{ Peso ao nascer } \\
\hline$<2.500 \mathrm{~g}$ & 72,7 & 1,137 & $0,938-1,377$ & 0,191 \\
\hline $2.500 \mathrm{~g}$ ou mais & 64,0 & 1 & & \\
\hline \multicolumn{5}{|l|}{ Idade da criança } \\
\hline 6 a 8 meses & 71,5 & 1,219 & $1,080-1,375$ & 0,001 \\
\hline 9 a 11 meses & 58,7 & 1 & & \\
\hline \multicolumn{5}{|l|}{ Internação hospitalar } \\
\hline Sim & 76,2 & 1,196 & $0,998-1,434$ & 0,052 \\
\hline Não & 63,7 & 1 & & \\
\hline \multicolumn{5}{|l|}{ Uso de chupeta } \\
\hline Sim & 64,6 & 1,002 & $0,888-1,132$ & 0,971 \\
\hline Não & 64,5 & 1 & & \\
\hline \multicolumn{5}{|l|}{ Uso de mamadeira } \\
\hline Sim & 64,3 & 0,991 & $0,877-1,120$ & 0,888 \\
\hline Não & 64,9 & 1 & & \\
\hline
\end{tabular}

Em Barra Mansa, quase dois terços das crianças no segundo semestre de vida recebiam alimentação não diversificada. Porém, esses resultados foram mais favoráveis do que os encontrados em vários estudos internacionais, que também adaptaram o indicador proposto pela $\mathrm{OMS}^{15-18}$, analisando quatro ou mais grupos de alimentos dos sete propostos pela OMS. Realizados com base em pesquisas da Demographic and Health Surveys (DHS), esses trabalhos encontraram uma prevalência de $82 \%$ no Nepal ${ }^{15}$ e de $80 \%$ em Bangladesh ${ }^{16}$ para a falta de diversidade alimentar. Valores ainda inferiores foram observados na Indonésia 
Tabela 4. Razão de prevalência ajustada $\left(\mathrm{RP}_{\mathrm{a}}\right)$ da ausência de diversidade alimentar no segundo semestre de vida, de acordo as características sociodemográficas e reprodutivas das mães e com os dados de nascimento e de assistência à criança, em de Barra Mansa, Sudeste do Brasil, 2006

\section{Características}

Acompanhante da criança

Mãe

Outro

Hospital de nascimento

Sistema Único de Saúde

Privado

Internação hospitalar

Sim

Não

Idade da criança em dias

$\mathbf{R P}_{\mathbf{a}}$

1

1,170

1

0,816

1,214

1

0,997
IC95\%

p-valor

$1,020-1,343$

0,025

0,001

$0,726-0,917$

$1,024-1,440$

0,026

0,996-0,999
$<0,001$
$(52 \%)^{17}$ e no Sri Lanka $(47 \%)^{18}$, denotando o quanto essa prática ainda é deficiente nos diversos cenários pesquisados. Contudo, um estudo em países da América Latina encontrou cenários heterogêneos quanto à diversidade alimentar de crianças entre 6 a 24 meses de idade, com consumo de cinco a sete grupos de alimentos (entre sete possíveis), variando de 65\% na Colômbia, $58 \%$ no Peru e $34 \%$ no Haiti ${ }^{12}$. No Rio de Janeiro, um estudo de 2006, que considerou a ausência de variância alimentar como o consumo de menos de quatro grupos de alimentos, constatou uma prevalência de $28 \%{ }^{8}$.

O presente artigo mostrou que $60,2 \%$ das crianças estavam sendo amamentadas, resultado inferior ao preconizado pela $\mathrm{OMS}^{3}$. No entanto, a prevalência encontrada foi semelhante à da Pesquisa Nacional de Demografia e Saúde da Criança e da Mulher no mesmo ano $(61,5 \%)^{19}$, o que reforça a validade interna dos dados obtidos. Outro achado foi o consumo de outros leites e de mingau por mais de um terço das crianças amamentadas, prática questionável, pois o leite materno provê, além dos nutrientes essenciais, uma ampla variedade de componentes bioativos não nutritivos que favorecem o crescimento infantil, de forma superior à composição das fórmulas infantis, que se limitam a sintetizar poucos componentes bioativos isolados ${ }^{20}$. As vantagens do leite materno não se limitam aos seus componentes nutricionais, como demonstrou um estudo longitudinal americano, no qual, ao final do primeiro ano de vida, crianças amamentadas obtiveram escores de desenvolvimento psicomotor, mental e de linguagem superiores aos daquelas alimentadas com fórmulas infantis à base de leite de vaca ou de soja ${ }^{21}$.

Em Barra Mansa, o consumo de frutas (ou suco de frutas) foi observado em quase $85 \%$ das crianças, resultado similar ao encontrado no segundo semestre de vida em outras cidades do Sudeste brasileiro ${ }^{8,22}$, mas inferior ao elevado consumo (97\%) em municípios do Nordeste ${ }^{23}$.

Quanto aos legumes, eles apresentaram elevado consumo em relação aos demais grupos pesquisados, porém cerca de um quinto das crianças no segundo semestre de vida não recebia esses alimentos, semelhante ao observado em cidades do Sudeste ${ }^{24}$ e do Sul25, mas superior do que três municípios de baixa renda (61\%) do Sudeste brasileiro ${ }^{26}$.

Entre os grupos alimentares estudados em Barra Mansa, o consumo de carne pelas crianças no segundo semestre de vida foi o menor, com menos de $60 \%$. Ainda assim, foi superior ao de um município do Nordeste $(34 \%)^{27}$, mas inferior ao encontrado em capital da região Sudeste $(75 \%)^{8}$. O uso desse grupo de alimentos (carne de boi, frango, peixe ou outros) na alimentação das crianças no segundo semestre de vida vem sendo pouco investigado, apesar de sua importância como fonte proteica e de ferro com alto valor biológico ${ }^{1}$. O baixo consumo pode ser devido a hábitos culturais da família ou à dificuldade de acesso a esse grupo alimentar pelo seu custo elevado em relação a outros alimentos.

O feijão, fonte de ferro, proteínas e carboidratos, foi consumido por mais de três quartos das crianças, de forma semelhante a uma cidade da região Norte do Brasil, onde $74 \%$ das crianças entre 6 e 8 meses e $86 \%$ daquelas entre 9 e 11 meses comiam feijão ${ }^{28}$, mas superior ao estudo realizado em 136 municípios de São Paulo, no qual o consumo foi de $58 \%{ }^{22}$.

$\mathrm{Na}$ presente pesquisa, a ausência de diversidade alimentar esteve diretamente associada à criança não estar acompanhada da mãe e à internação hospitalar prévia, enquanto o financiamento privado do hospital para o nascimento e a idade crescente da criança em dias associaram-se inversamente ao desfecho.

A criança não ter vindo acompanhada de sua mãe para ser vacinada foi um fator associado à falta de diversidade alimentar, com prevalência 17\% superior no segundo semestre de vida. Possivelmente, crianças não acompanhadas para um cuidado à saúde, como a imunização, podem ter a mãe menos presente também para outros cuidados, recebendo, assim, uma alimentação menos diversificada, mas essa variável precisa ser mais bem explorada em estudos futuros.

A internação hospitalar prévia esteve associada a uma prevalência $21 \%$ superior ao desfecho, mas não foi possível estabelecer se ela foi responsável pela modificação do padrão alimentar da criança ou se a falta de diversidade alimentar 
contribuiu para a internação (causalidade reversa). A criança que ficou internada pode ter modificado a alimentação porque ficou debilitada e sem apetite. Vale ressaltar que um dos passos para uma alimentação saudável é oferecer à criança doente e convalescente seus alimentos habituais, preferidos e diversificados, respeitando a sua aceitação ${ }^{29}$.

As crianças que nasceram em hospital privado tiveram uma alimentação complementar mais variada, possivelmente porque as mães tinham uma condição socioeconômica que favorecia a aquisição de alimentos diversificados, incluindo o grupo das carnes, de custo mais elevado.

A idade crescente da criança em dias apresentou-se diretamente associada à alimentação diversificada, de forma semelhante à encontrada em Bangladesh ${ }^{16}$, na Indonésia ${ }^{17}$ e no Sri Lanka ${ }^{18}$. Outro estudo de coorte multicêntrico realizado em Shangai, Cincinnati e Cidade do México, com crianças entre 6 e 12 meses de idade, observou que a variação alimentar aumenta com a idade crescente da criança ${ }^{30}$. Esse resultado é importante, pois um suprimento adequado de nutrientes é cada vez mais necessário para o crescimento da criança a partir dos 6 meses $^{2}$.
Algumas limitações do presente estudo devem ser ressaltadas. Pesquisas em campanhas de vacinação permitem a obtenção de informações em um curto período e com um custo baixo, mas estudos seccionais, em geral, não possibilitam explorar a relação de temporalidade entre os fatores de exposição e o desfecho. Os resultados do estudo não puderam ser comparados à literatura nacional pela pouca produção acadêmica relativa a esse tema. Além disso, a OMS não estabeleceu pontos de corte que permitam definir o grau de adequação das práticas alimentares.

No contexto brasileiro, este é o primeiro trabalho a analisar fatores associados à falta de diversidade alimentar de lactentes no segundo semestre de vida. O monitoramento dessas práticas faz-se importante para identificar o impacto das políticas públicas voltadas para alimentação complementar saudável e para que grupos de risco recebam mais atenção pelos serviços de saúde. Recomenda-se que as políticas públicas de alimentação complementar saudável ${ }^{29}$ sejam intensificadas em conjunto com estratégias de outras esferas de ação, como as políticas econômicas de distribuição de renda, no intuito de melhorar essas práticas.

\section{REFERÊNCIAS}

1. Monte CMG, Giugliani ERJ. Recommendations for the complementary feeding of the breastfed child. J Pediatr. 2004;80(5 Suppl):131-41. http:// dx.doi.org/10.1590/S0021-75572004000700004. PMid:15583763.

2. Pan American Health Organization. World Health Organization. Guiding principles for complementary feeding of the breastfed child. Washington: PAHO; Geneva: WHO; 2003.

3. World Health Organization. Infant and young child nutrition: global strategy on infant and young child feeding. Geneva: WHO; 2002. N ${ }^{\circ}$. Resolution WHA55/15.

4. World Health Organization. Indicators for assessing infant and young child feeding practices: part 1 definitions. Geneva: WHO; 2008.

5. Steyn NP, Nel JH, Nantel G, Kennedy G, Labadarios D. Food variety and dietary diversity scores in children: are they good indicators of dietary adequacy? Public Health Nutr. 2006;9(5):644-50. http://dx.doi.org/10.1079/ PHN2005912. PMid:16923296.

6. Instituto Brasileiro de Geografia e Estatística. IBGE cidades [Internet]. Rio de Janeiro: IBGE; 2015 [citado em 2015 set 3]. Disponível em: http:// www.cidades.ibge.gov.br

7. Brasil. Ministério da Saúde. II Pesquisa de Prevalência de Aleitamento Materno nas capitais brasileiras e Distrito Federal. Brasília: Ministério da Saúde; 2009.

8. Oliveira DA, Castro IRR, Jaime PC. Complementary feeding patterns in the first year of life in the city of Rio de Janeiro, Brazil: time trends from 1998 to 2008. Cad Saude Publica. 2014;30(8):1755-64. http://dx.doi. org/10.1590/0102-311X00120013. PMid:25210914.

9. Coutinho LMS, Scazufca M, Menezes PR. Methods for estimating prevalence ratios in cross-sectional studies. Rev Saude Publica. 2008;42(6):992-8. http://dx.doi.org/10.1590/S0034-89102008000600003. PMid:19009156.
10. Brasil. Ministério da Saúde. Conselho Nacional de Saúde. Resolução no 196, de 10 de outubro de 1996. Aprova as seguintes diretrizes e normas regulamentadoras de pesquisas envolvendo seres humanos. Diário Oficial da União, Brasília, 1996.

11. Birch LL. Conducta alimentar en los niños: perspectiva de su desarrollo. In: Organización Panamericana De La Salud. Nutrición y alimentación del niño en los primeros años de vida. Washington: OPS; 1997. p. 34-48.

12. Arimond M, Ruel MT. Dietary diversity is associated with child nutritional status: evidence from 11 Demographic and Health Surveys. J Nutr. 2004;134(10):2579-85. http://dx.doi.org/0022-3166/04. PMid:15465751.

13. Ramos M, Stein LM. Development children's eating behavior. J Pediatr. 2000;76(Suppl 3):S229-37. http://dx.doi.org/0021-7557/00/76-Supl.3/S229. PMid:14676901.

14. Vieira GO, Silva LR, Vieira TO, Almeida JAG, Vilma AC. Feeding habits of breastfed and non-breastfed children up to 1 year old. J Pediatr. 2004;80(6):411-6. http://dx.doi.org/10.2223/JPED.1227. PMid:15505738.

15. Joshi N, Agho KE, Dibley MJ, Senarath U, Tiwari K. Determinants of inappropriate complementary feeding practices in young children in Nepal: secondary data analysis of Demographic and Health Survey 2006. Matern Child Nutr. 2012;8(Suppl 1):45-59. http://dx.doi.org/10.1111/j.17408709.2011.00384.x. PMid:22168518.

16. Kabir I, Khanam M, Agho KE, Mihrshahi S, Dibley MJ, Roy SK. Determinants of inappropriate complementary feeding practices in infant and young children in Bangladesh: secondary data analysis of Demographic Health Survey 2007. Matern Child Nutr. 2012;8(Suppl 1):11-27. http://dx.doi. org/10.1111/j.1740-8709.2011.00379.x. PMid:22168516.

17. Ng CS, Dibley MJ, Agho KE. Complementary feeding indicators and determinants of poor feeding practices in Indonesia: a secondary analysis of 2007 demographic and health survey. Public Health Nutr. 2011;15(5):82739. http://dx.doi.org/10.1017/S1368980011002485. PMid:22014663. 
18. Senarath U, Godakandage SSP, Jayawickrama H, Siriwardena I, Dibley MJ. Determinants of inappropriate complementary feeding practices in young children in Sri Lanka: secondary data analysis of Demographic and Health Survey 2006-2007. Matern Child Nutr. 2012;8(Suppl 1):60-77. http://dx.doi. org/10.1111/j.1740-8709.2011.00370.x. PMid:22168519.

19. Brasil. Ministério da Saúde. PNDS 2006: Pesquisa Nacional de Demografia e Saúde da Criança e da Mulher. Brasília: Ministério da Saúde; 2008.

20. Ballard O, Morrow AL. Human milk composition: nutrients and bioactive factors. Pediatr Clin North Am. 2013;60(1):49-74. http://dx.doi.org/10.1016/j. pcl.2012.10.002. PMid:23178060.

21. Andres A, Cleves MA, Bellando JB, Pivik RT, Casey PH, Badger TM. Developmental status of 1-year-old infants fed breast milk, cow's milk formula, or soy formula. Pediatrics. 2012;129(6):1134-40. PMid:22641754.

22. Saldiva SR, Escuder MM, Mondini L, Levy RB, Venancio SI. Feeding habits of children aged 6 to 12 months and associated maternal factors. J Pediatr. 2007;83(1):53-8. http://dx.doi.org/10.2223/JPED.1588. PMid:17279291.

23. Caetano MC, Ortiz TT, da Silva SG, de Souza FI, Sarni RO. Complementary feeding: inappropriate practices in infants. J Pediatr. 2010;86(3):196-201. http://dx.doi.org/10.1590/S0021-75572010000300006. PMid:20401426.

24. Cruz MCC, Almeida JAG, Engstrom EM. Praticas alimentares no primeiro ano de vida de filhos de adolescentes. Rev Nutr. 2010;23(2):201-10. http:// dx.doi.org/10.1590/S1415-52732010000200003.

25. Bercini LO, Masukawa MLT, Martins MR, Labegalini MPC, Alves NB. Alimentação da criança no primeiro ano de vida, em Maringá, PR. Ciênc
Cuid Saúde. 2007;6(2):404-10. http://dx.doi.org/10.4025/cienccuidsaude. v6i0.5339.

26. Silveira FJF, Lamounier JA. Prevalência do aleitamento materno e práticas de alimentação complementar em crianças com até 24 meses de idade na região do Alto do Jequitinhonha, Minas Gerais. Rev Nutr. 2004;17(4):43747. http://dx.doi.org/10.1590/S1415-52732004000400004.

27. Oliveira LPM, Assis AMO, Pinheiro SMC, Prado MS, Barreto ML. Alimentação complementar nos primeiros dois anos de vida. Rev Nutr. 2005;18(4):459-69. http://dx.doi.org/10.1590/S1415-52732005000400002.

28. Garcia MT, Granado FS, Cardoso MA. Alimentação complementar e estado nutricional de crianças menores de dois anos atendidas no Programa Saúde da Família em Acrelândia, Acre, Amazônia Ocidental Brasileira. Cad Saude Publica. 2011;27(2):305-16. http://dx.doi.org/10.1590/S0102311X2011000200012. PMid:21359467.

29. Brasil. Ministério da Saúde. Secretaria de Atenção à Saúde. Departamento de Atenção Básica. Dez passos para uma alimentação saudável: Guia alimentar para crianças menores de dois anos: um guia para o profissional da saúde na atenção básica. 2 ed. Brasília: Ministério da Saúde; 2013.

30. Woo JG, Herbers PM, McMahon RJ, Davidson BS, Ruiz-Palacios GM, Peng YM, et al. Longitudinal development of infant complementary diet diversity in 3 international cohorts. J Pediatr. 2015;167(5):969-974.e1. http://dx.doi.org/ 10.1016/j.jpeds.2015.06.063.

Recebido em: Dez. 22, 2016 Aprovado em: Mar. 07, 2017 